\title{
Human CD4+ Memory T-cell Populations Secrete Th1 Cytokines in Response to Influenza Antigen Stimulation
}

\section{Shan Chen ${ }^{1 \dagger}$, David Roumanes ${ }^{2 \dagger}$, Maria T. Arevalo ${ }^{1}$, Yanping Chen ${ }^{1}$ and Mingtao Zeng ${ }^{1,2 *}$}

${ }^{1}$ The Center of Excellence for Infectious Diseases, Department of Biomedical Sciences, Paul L. Foster School of Medicine, Texas Tech University Health Sciences Center, EI Paso, Texas, USA

${ }^{2}$ The Department of Microbiology \& Immunology, School of Medicine and Dentistry, University of Rochester, Rochester, New York, USA

these two authors are equal contributors

\begin{abstract}
Seasonal influenza vaccine efficacy is measured by the vaccine's ability to elicit strain-specific antibody responses. Every year, resources are allocated into formulating new influenza vaccines. Candidate vaccines utilizing memory T-cells may afford long-term protection. Our study characterizes the human CD4+ memory T-cell response to influenza virus. Intracellular cytokine staining assay was used to assess T-cell production of several cytokines (IFN- $y$, IL-2, TNF- $\alpha$, IL-4, IL-5, and IL-17) found in human peripheral blood mononuclear cells after stimulation with influenza antigens. Production of Th1 cytokines (IFN- $\gamma$, TNF- $\alpha$, and IL-2) was significant in activated CD4+ T-cells after stimulation, whereas Th2 cytokine secretion remained unchanged. In addition, a significant increase in multifunctional CD4+ T-cells that simultaneously secreted combinations of IL-2, IFN- $\gamma$, and TNF- $\alpha$ was observed. Our studies have revealed that CD4+ T-cell responses against influenza are Th1-biased, raising the possibility of identifying these populations as targets for successful influenza vaccination.
\end{abstract}

Keywords: Influenza; CD4+; Memory; T Cell; Cytokines

\section{Introduction}

Influenza is an important health issue due to the emergence of several pandemic strains during the past century: 1918 H1N1, 1957 H2N2, $1968 \mathrm{H} 3 \mathrm{~N} 2$ and $2009 \mathrm{H} 1 \mathrm{~N} 1$ [1]. Current licensed vaccines protect health by eliciting antibody responses against the viral surface glycoproteins hemagglutinin (HA) and neuraminidase (NA). However, influenza viruses evade host memory against $\mathrm{HA}$ and NA via antigenic drift and shift. Thus, vaccines are prepared annually containing three influenza strains predicted to be prevalent in the upcoming season [2]. Upon identification of a possible pandemic strain, it may take six months to develop a new vaccine [3]. The current licensed approach is insufficient.

New candidate vaccines attempt to address these issues by targeting conserved epitopes including influenza matrix protein 1 (M1), matrix protein 2 (M2), and nucleoprotein (NP) to elicit long-term protection. Humoral immunity prevents the spread of virus, while T-cell immunity mediates viral clearance. T-cell immunity against conserved proteins is heterosubtypic [4], reacting with multiple strains of influenza virus. M1, M2, and NP-specific T-cell epitopes have all been shown to be cross-strain reactive [5-8]. Furthermore, T-cell responses to M1 and NP are not only cross-reactive, but also immunodominant [7]. Therefore, influenza vaccines should be tested for their ability to elicit T-cell immunity.

The CD4+ T-cell is of interest to our study. While heterosubtypic immunity to influenza is mostly mediated by CD8+ cytotoxic T-cells [9], CD4+ T-cell help is critical in generating functional CD8+ T-cell memory [10], in influencing B-cells to differentiate into antibodysecreting plasma cells [11], and in regulating the innate inflammatory response [12]. Furthermore, CD4+ T-cells dictate CD8+ responses via secretion of IFN- $\gamma$, IL-2, and TNF- $\alpha$ [13].

CD4+ T-cells can be classified based on polarization. T helper 1 (Th1) cells will elicit a strong IFN- $\gamma$ response; Th 2 cells are characterized by IL-4 secretion; Th17 secrete IL-17, which enhances the inflammatory response; and regulatory T-cells (Treg) produce IL-10 and TGF- $\beta$ to maintain peripheral tolerance and dampen immune responses [14].
In humans, stimulation of memory T-cells leads to increased IFN- $\gamma$ $[15,16]$ secretion by CD4+ cells.

Primary literature describing human memory CD4+ T-cell responses to influenza are relatively scarce. In our study, we utilized a complex multiparametric 14-color flow cytometry analysis to detect human influenza-specific memory T-cell responses. The scope of our study encompassed many aspects of CD4+ T-cell responses, testing for activated Th1, Th2, Th17, and T-regulatory cell subsets.

\section{Materials and Methods}

\section{Peripheral blood mononuclear cell isolation and antigenic stimulation}

Healthy human volunteers between the ages of 25 to 58 were enrolled for a single visit study after approval by the institutional ethics committee. Each volunteer provided written, informed consent in accordance with the principles of the Helsinki Declaration of 1975. Patient information on age, gender, and influenza vaccination status was collected (Table 1). $30 \mathrm{ml}$ of blood was collected in $10 \mathrm{U} /$ $\mathrm{ml}$ heparin from each of seven donors. Peripheral blood mononuclear cells (PBMCs) were isolated using lymphocyte separation medium (Invitrogen, Carlsbad, CA) as per protocol and cryopreserved in liquid nitrogen. Isolated cells were thawed and washed twice in RPMI medium (Invitrogen) supplemented with 8\% fetal bovine serum (Invitrogen), 2

*Corresponding author: Mingtao Zeng, Ph.D./Associate Professor, Center of Excellence for Infectious Diseases, Department of Biomedical Sciences, Paul L. Foster School of Medicine, Texas Tech University Health Sciences Center, 5001 El Paso Drive, El Paso, Texas 79905, USA, Tel: +1 9157831241 ext 253; Fax: +1 915 7831271; E-mail: mt.zeng@ttuhsc.edu

Received March 14, 2012; Accepted March 23, 2012; Published March 25, 2012

Citation: Chen S, Roumanes D, Arevalo MT, Chen Y, Zeng M (2012) Human CD4+ Memory T-cell Populations Secrete Th1 Cytokines in Response to Influenza Antigen Stimulation. J Vaccines Vaccin 3:129. doi:10.4172/2157-7560.1000129

Copyright: () 2012 Chen S, et al. This is an open-access article distributed under the terms of the Creative Commons Attribution License, which permits unrestricted use, distribution, and reproduction in any medium, provided the original author and source are credited. 
Citation: Chen S, Roumanes D, Arevalo MT, Chen Y, Zeng M (2012) Human CD4+ Memory T-cell Populations Secrete Th1 Cytokines in Response to Influenza Antigen Stimulation. J Vaccines Vaccin 3:129. doi:10.4172/2157-7560.1000129

Page 2 of 4

\begin{tabular}{lcccc}
\hline Donation Date & Age & Gender & Vaccinated (Y/N) & Vaccination date \\
\hline $1 / 12 / 2009$ & 25 & F & no & n/a \\
$1 / 13 / 2009$ & 37 & M & no & n/a \\
$1 / 13 / 2009$ & 28 & M & yes & $10 / 2008$ \\
$1 / 14 / 2009$ & 28 & M & no & n/a \\
$1 / 19 / 2009$ & 58 & M & yes & Fall 2008 \\
$1 / 20 / 2009$ & 42 & F & yes & $10 / 2008$ \\
$1 / 21 / 2009$ & 34 & M & yes & $11 / 2008$ \\
\hline
\end{tabular}

Vaccination in 2008 was with the seasonal trivalent vaccine containing $15 \mu \mathrm{g} \mathrm{HA}$ $\mathrm{n} / \mathrm{a}=$ not applicable

Table 1: Age, Gender, and Vaccination Status of Healthy Donors.

$\mathrm{mM}$ glutamine (Invitrogen), 1\% penicillin/streptomycin (Invitrogen), and $10 \mu \mathrm{g} / \mathrm{ml}$ DNase (Invitrogen). Cells were then cultured in a $25 \mathrm{~cm}^{2}$ flask at $2 \times 10^{6}$ cells $/ \mathrm{mL}$ (same medium) at $37^{\circ} \mathrm{C}$ and $5 \% \mathrm{CO}_{2}$ overnight. Cell number and viability were assessed using Trypan Blue (Sigma, St. Louis, MO) exclusion. PBMCs were then transferred to a 96-well U-bottom culture plate, exposed to $0.1 \mu \mathrm{g}$ of influenza antigens, and incubated at $37^{\circ} \mathrm{C}$ and $5 \% \mathrm{CO}$ for 2 hours. The 2008-2009 FluZone pediatric influenza vaccine is a split-virion vaccine composed of $\mathrm{A} /$ Brisbane/59/2007 (H1N1), A/Uruguay/716/2007 (H3N2), and B/ Florida/04/2006 influenza viral strains and was used as the source of influenza antigens. Each $0.25 \mathrm{ml}$ dose of FluZone pediatric vaccine contains a combined total of $22.5 \mu \mathrm{g}$ of HA. Cells were also stimulated in parallel and for the same duration with $1 \mu \mathrm{g} / \mathrm{mL}$ staphylococcal enterotoxin B (SEB) (Sigma) as a positive control and with medium alone as a negative control. Cells were then treated with $2 \mu \mathrm{M}$ Monensine and $10 \mu \mathrm{g} / \mathrm{ml}$ Brefeldine A and incubated at $37^{\circ} \mathrm{C}$ and $5 \%$ $\mathrm{CO}_{2}$ for 8 hours. The preparations were refrigerated at $4^{\circ} \mathrm{C}$ overnight.

\section{Immunofluorescence staining}

Cell samples were washed twice with PBS supplemented with $2 \%$ human serum (staining buffer) and stained for $30 \mathrm{~min}$ at $4^{\circ} \mathrm{C}$ for surface antigens. Monoclonal antibodies against human CD4-APCAlexa 750 (eBioscience, San Diego, CA), CD8-QDot 565 (Invitrogen), CD14-QDot 800 (Invitrogen), CD25-APC (BD Biosciences, San Jose, CA), CD45RA- QDot 655 (Invitrogen), and CD56- PE-Cy5-5 (Invitrogen) were used. For intracellular staining, the cells were fixed and permeablized for 20 minutes at $4^{\circ} \mathrm{C}$ using BD Cytofix/Cytoperm (BD Biosciences) and stained for 30 minutes at $4^{\circ} \mathrm{C}$ with monoclonal antibodies purchased from Invitrogen or BD Biosciences: QDot 605 labeled CD3, PE-Cy5 labeled CD69, PerCp-Cy5-5 labeled TNF- $\alpha$, PECy7 labeled IFN- $\gamma$, Alexa 700 labeled IL-2, PE labeled IL-4/IL-5, Pacific Blue labeled IL-17A, and Alexa 488 labeled FoxP3. Finally, the cells were washed twice and resuspended in $300 \mu$ staining buffer.

\section{Flow cytometry and analyses}

14-color flow cytometric data were collected on a BD LSRII instrument (BD Biosciences) and analyzed using FlowJo software (TreeStar, Ashland, OR). Dead cells were excluded by forward and side scatter gating. A quadrant gating method was used (Figure 1); analyses were standardized. Samples were acquired consecutively. CD4+ T-cell populations were separated based on the following attributes: CD3+ (T-cell co-receptor), CD4+ (expressed on Th cells, Treg cells, monocytes, macrophages, natural killer cells, and dendritic cells), CD8(expressed mainly on cytotoxic T-cells and natural killer cells), CD14(expressed on monocytes, macrophages, neutrophils, and dendritic cells), CD45RA- (expressed on naïve T-cells), CD56- (expressed on natural killer cells), and CD69+ (expressed in activated T-cells). This population was analyzed for the expression of additional parameters: IFN- $\gamma$, IL-2, TNF- $\alpha$, IL-17A, CD25, FoxP3, IL-4, and IL-5. Percentages were based on the ratio of cytokine secreting cells (CD3+/CD4+/CD8-/ CD14-/CD45RA-/CD56-/CD69+/cytokine) to total CD4+ T-cell (CD3+/CD56-/CD14-/CD8-/CD4+) populations.

\section{Statistical analyses}

Mann-Whitney tests were used to compare antigen-stimulated samples to medium-only stimulated (negative) samples using Prism 5.04 software (GraphPad, La Jolla, CA). P $<0.05\left(^{*}\right)$ was considered significant.

\section{Results}

\section{Influenza specific memory CD4+ T-cell response is Th1-} biased

Influenza-specific CD4+ memory T-cells in PBMC culture were characterized by IFN- $\gamma$, TNF- $\alpha$, and IL- 2 secretion upon activation (CD45RA-CD69+, Figure 2). Representative dot plots are shown for samples that were stimulated with medium-only (NEG), FluZone (FLU), or SEB as a positive control (Figure 2A). These influenzaspecific, memory CD4+ T cells significantly expressed IFN $-\gamma$, TNF- $\alpha$, and IL-2 $(8.40 \%, p=0.006 ; 13.63 \%, p=0.0104$; and $4.76 \% . p=0.0021$, respectively, Figure 2B) as compared to PBMC stimulated with medium-only (NEG). Additional analyses compared the frequency of these cytokine secreting cells from vaccinated $(n=4)$ versus unvaccinated donors $(n=3)$ and detected no significant differences.

\section{Detection of influenza-specific multifunctional CD4+ T-cells}

Simultaneous production of IFN- $\gamma$ and IL-2; TNF- $\alpha$ and IL2 ; IFN- $\gamma$ and TNF- $\alpha$; and IFN- $\gamma$, TNF- $\alpha$, and IL-2 (Figure 2C) in influenza-stimulated and activated $\mathrm{CD} 4+\mathrm{T}$-cells was evaluated. Significant and distinct populations of influenza-specific IFN- $\gamma+/ \mathrm{IL}-$ $2+(6.64 \%, p=0.0021)$, TNF- $\alpha+/$ IL-2+ (8.25\%, $p=0.0006)$, IFN $-+/$ TNF- $\alpha+(8.84 \%, p=0.0006)$, and IFN- $\gamma+/$ TNF- $\alpha+/$ IL-2 (5.63\%, $p=$ $0.0021)$ were detected in PBMC.
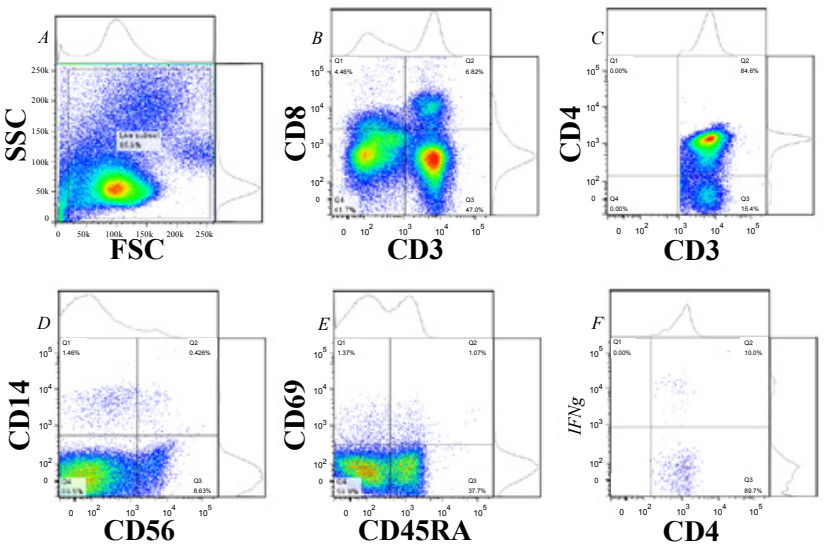

Figure 1: Quadrant gating method. Representative dot plots are shown for the gating strategy used. A: Live cells were selected by a square gate in the forward and side scatter plot as to exclude anomalous cell particles. B: CD3+ and CD8- populations were selected. C: CD4+ populations were selected. D: Then, CD56- and CD14- populations were selected. This population number (CD3+/CD8-/CD4+/CD56-/CD14-) was used as the total CD4+ T-cell count E: CD45RA- and CD69+ populations were selected. F: Various cytokines and other markers (such as IFNy) were plotted against CD4+ and cell population percentages were used for data analyses. 
A

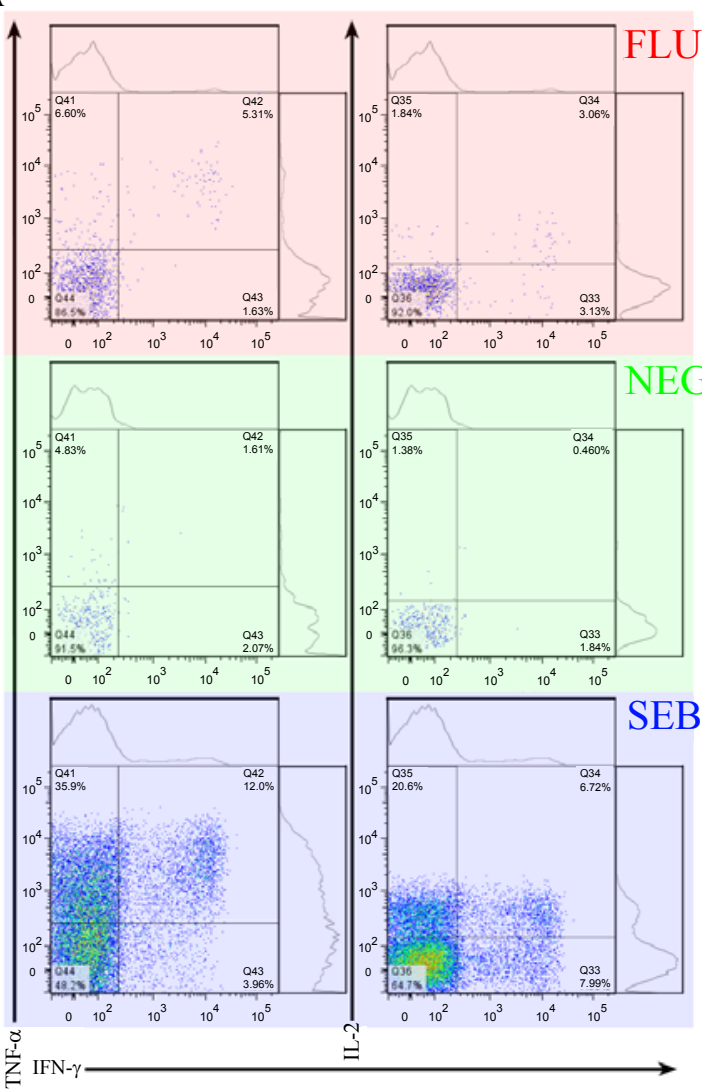

B
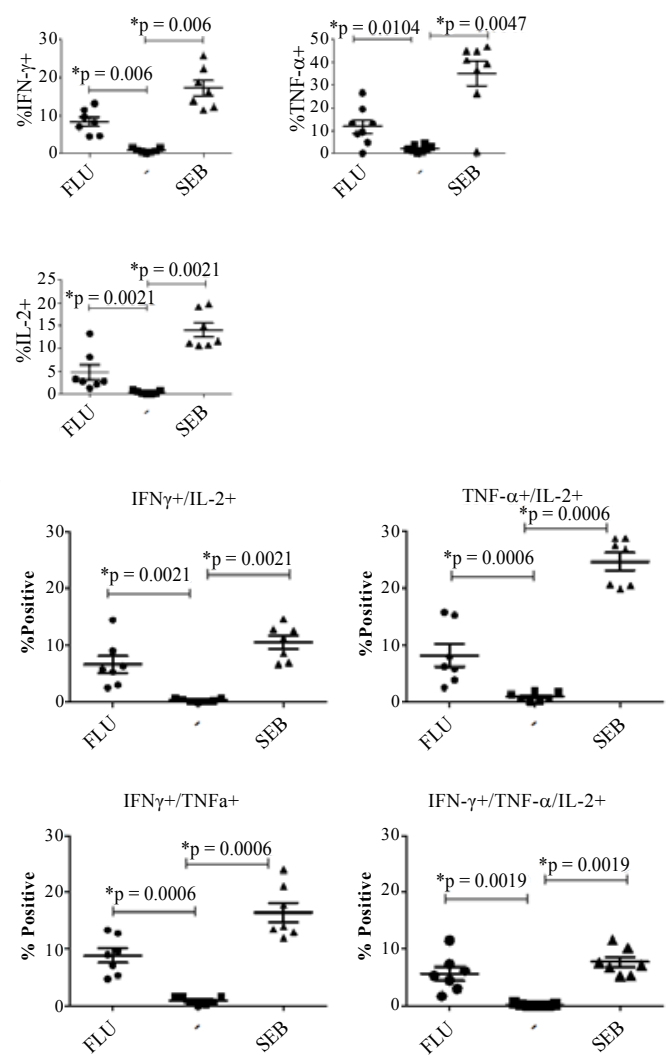

Figure 2: Th1 response by influenza-specific memory CD4+ cells. Activated human CD4+ memory cells (CD3+CD4+CD8-CD14-CD45RA-CD56-CD69+) were analyzed for expression of IFN- $\gamma$, TNF- $\alpha$, and IL-2. Cells were stimulated with medium-only (NEG), FluZone vaccine (three influenza strains at equivalent concentrations based on viral HA), or SEB. A: Representative density plots with histograms are shown for activated memory CD4+ cells expressing IFN-y (increasing from left to right), TNF- $\alpha$ (left column, increasing from bottom to top), and IL-2 (right column, increasing from bottom to top). Percentages are based on total CD4+ T-cells in each sample and results are shown as the mean \pm standard error (SE) $(n=7)$. B: Single cytokine populations IFN- $\gamma$, TNF- $\alpha$, and IL-2 are shown. C: Double and triple cytokine expressing (IFN- $/$ /IL-2, TNF- $\alpha /$ IL-2, IFN- $/$ TNF- $\alpha$, IFN- $/$ /TNF- $\alpha /$ IL-2) CD4+ T-cell populations are shown. Stimulated samples were compared to their unstimulated counterparts using Mann-Whitney statistical tests.

\section{Th2, Th17, and T-regulatory CD4+ T-cell subsets did not respond to antigen stimulation}

Stimulation with influenza antigens did not induce Th2 cytokines IL-4 and IL-5 (not shown, antigen stimulated cells did not exceed medium-only stimulated cells), and IL-17 production was not significantly increased after stimulation (not shown, $p=1.0000$ ). Also, stimulation did not increase the percentage of T-regulatory as defined by CD25+/FoxP3+ expression (not shown, $p=0.5224$ ).

\section{Discussion}

In this study, we characterized the small subset of activated memory CD4+ T-cells upon stimulation with the pediatric trivalent FluZone vaccine. Previously, it has been difficult to study memory CD4+ T-cells due to low numbers that persist after influenza infection [13]. Our method is sensitive enough to reveal a robust induction of Th1 cytokines (IFN- $\gamma$, TNF- $\alpha$, and IL-2) in memory CD4+ T-cells. IFN- $\gamma$ promotes suppression of Th2 cell activity, Th1 differentiation via T-bet and increased expression of class I MHC molecules [17]. TNF- $\alpha$ is a pleiotropic cytokine with functions in the inflammatory response, apoptosis, and survival [18,19]. IL-2 is responsible for T-cell proliferation [20]. Thus, not only is it possible that memory CD4+
T-cells may play a role in inflammatory and antiviral responses to influenza, but they may also function in modulating the responses of other immune cells [13].

Upon further analysis, the data revealed significant $(\mathrm{p}<0.05)$ multifunctional populations (IFN- $\gamma+/ \mathrm{IL}-2+$, TNF- $\alpha+/ \mathrm{IL}-2+$, IFN- $\gamma+/$ TNF- $\alpha+$, and IFN- $\gamma+/$ TNF- $\alpha+/$ IL- $2+$ ) in the activated, memory CD4+ compartment, in accordance with other studies [21]. The frequency of multifunctional IFN- $\gamma+/$ TNF- $\alpha+/$ IL- $2+$ T-cells has been shown to correlate with immunogenicity and protection rendered against Mycobacterium tuberculosis [22], Chlamydia muridarum [23], and HIV-1 [11] by their respective vaccine candidates. In agreement, our results show that these populations can be strong clinical indicators of immunological memory. Thus, understanding of these multifunctional $\mathrm{T}$-cell populations may provide insights into both the design and assessment of future influenza vaccines.

We did not detect influenza-specific Th2 (IL-4/IL-5) or Th17 (IL-17) cytokines in this study. While our stimulation time may be viewed as short, it has been shown that accessory-cell stimulation of IL-4 secretion by human T-cells in the presence of Brefeldin A peaks between 0-6 hours [24,25]. Moreover, in choosing an appropriate stimulation time, we took into account that multifunctional subsets 
are short-lived, with expression at a maximum between 0-6 hours, and disappear by 18-22 hours of stimulation [24]. We also did not detect influenza-specific T-regulatory cells (CD25+/FoxP3+ phenotype). However, as this initial study was modest $(n=7)$, we cannot definitively rule out the contribution to influenza memory immunity by these cell subsets.

In our donor pool, there were 4 donors who had received the seasonal influenza vaccine (2008-2009), and 3 donors that had not. We investigated if influenza-specific memory CD4+ responses differed. Statistically, there were no differences, which may be attributed to 1) sample size and 2) exposure to similar influenza strains, or 3) inability of the seasonal vaccine to significantly influence the cellular immune response. Recently, it has been shown that even live attenuated influenza vaccines fail to boost the cellular immune response in healthy adult subjects [26]. However, due to the small sample sizes, no meaningful conclusions can be derived.

Herein, we describe influenza specific CD4+ T-cells in the memory compartment. The response by these $\mathrm{CD} 4+\mathrm{T}$-cells derived from PBMCs was Th1-driven, producing IFN- $\gamma$, TNF- $\alpha$, and IL- 2 cytokines, but was, more importantly, multifunctional. Multifunctional, influenza-specific Th1 cells have previously been described in mice in the context of vaccine evaluations $[27,28]$ and in resident memory T-cells from human lungs [29], and thus our study identifies additional subsets of multifunctional, human Th1 memory cells found in human peripheral blood that may function in host defense against influenza in healthy adults.

\section{Acknowledgments}

This work was supported by the U.S. Public Service research grant AI072139 (M.Z.) from the National Institute of Allergy and Infectious Diseases, and the interna research fund from Texas Tech University Health Sciences Center Paul L. Foster School of Medicine and University of Rochester School of Medicine and Dentistry (M.Z.). We thank Tim Mossman and Sally Quataert for sharing their intracellular cytokine staining protocols. We greatly appreciate the effort of Deanna Maffett for recruiting study volunteers and collecting blood samples.

\section{References}

1. Belser JA, Maines TR, Tumpey TM, Katz JM (2010) Influenza A virus transmission: contributing factors and clinical implications. Expert Rev Mol Med 12: e39.

2. Seasonal Influenza (Flu) - Key Facts About Seasonal Flu Vaccine.

3. Global Alert and Response (GAR): Pandemic influenza vaccine manufacturing process and timeline.

4. Dutton RW, Swain SL, Woodland DL (2007) Vaccines against pandemic influenza. Viral Immunol 20: 326-327.

5. Thueng-in $\mathrm{K}$, Maneewatch $\mathrm{S}$, Srimanote $\mathrm{P}$, Songserm $\mathrm{T}$, Tapchaisri $\mathrm{P}$, et al (2010) Heterosubtypic immunity to influenza mediated by liposome adjuvanted H5N1 recombinant protein vaccines. Vaccine 28: 6765-6777.

6. Uchida T (2011) Development of a cytotoxic T-lymphocyte-based, broadly protective influenza vaccine. Microbiol Immunol 55: 19-27.

7. Lee LY, Ha do LA, Simmons C, de Jong MD, Chau NV, et al. (2008) Memory $T$ cells established by seasonal human influenza $A$ infection cross-react with avian influenza A (H5N1) in healthy individuals. J Clin Invest 118: 3478-3490.

8. Song JM, Wang BZ, Park KM, Van Rooijen N, Quan FS, et al. (2011) Influenza virus-like particles containing M2 induce broadly cross protective immunity. PLoS One 6: e14538.
9. Brown LE, Kelso A (2009) Prospects for an influenza vaccine that induces cross-protective cytotoxic T Iymphocytes. Immunol Cell Biol 87: 300-308.

10. Shedlock DJ, Shen H (2003) Requirement for CD4 T cell help in generating functional CD8 T cell memory. Science 300: 337-339.

11. Arthur RP, Mason D (1986) T cells that help B cell responses to soluble antigen are distinguishable from those producing interleukin 2 on mitogenic or allogeneic stimulation. J Exp Med 163: 774-786.

12. McKinstry KK, Strutt TM, Swain SL (2011) Hallmarks of CD4 T cell immunity against influenza. J Intern Med 269: 507-518.

13. Brown DM, Roman E, Swain SL (2004) CD4 T cell responses to influenza infection. Semin Immunol 16: 171-177.

14. Strutt TM, McKinstry KK, Swain SL (2009) Functionally diverse subsets in CD4 T cell responses against influenza. J Clin Immunol 29: 145-150.

15. Leroux-Roels I, Van der Wielen M, Kafeja F, Vandermeulen C, Lazarus R, et al (2009) Humoral and cellular immune responses to split-virion H5N1 influenza vaccine in young and elderly adults. Vaccine 27: 6918-6925.

16. Crowe BA, Bruhl P, Gerencer M, Schwendinger MG, Pilz A, et al. (2010) Evaluation of the cellular immune responses induced by a non-adjuvanted inactivated whole virus $\mathrm{A} / \mathrm{H} 5 \mathrm{~N} 1 / \mathrm{VN} / 1203$ pandemic influenza vaccine in humans. Vaccine 29: 166-173.

17. Boehm U, Klamp T, Groot M, Howard JC (1997) Cellular responses to interferon-gamma. Annu Rev Immunol 15: 749-795.

18. Vilcek J, Lee TH (1991) Tumor necrosis factor. New insights into the molecular mechanisms of its multiple actions. J Biol Chem 266: 7313-7316.

19. Rangamani $P$, Sirovich $L$ (2007) Survival and apoptotic pathways initiated by TNF-alpha: modeling and predictions. Biotechnol Bioeng 97: 1216-1229.

20. Smith KA (1988) Interleukin-2: inception, impact, and implications. Science 240: 1169-1176.

21. Mahnke YD, Saqr A, Hazenfeld S, Brady RC, Roederer M, et al. (2011) Agerelated changes in durability and function of vaccine-elicited influenza-specific CD4(+) T-cell responses. Vaccine 29: 8606-8614.

22. Derrick SC, Yabe IM, Yang A, Morris SL (2011) Vaccine-induced antituberculosis protective immunity in mice correlates with the magnitude and quality of multifunctional CD4 T cells. Vaccine 29: 2902-2909.

23. Yu H, Karunakaran KP, Kelly I, Shen $C$, Jiang X, et al. (2011) Immunization with Live and Dead Chlamydia muridarum Induces Different Levels of Protective Immunity in a Murine Genital Tract Model: Correlation with MHC Class I Peptide Presentation and Multifunctional Th1 Cells. J Immunol 186: 3615-3621.

24. Picker LJ, Singh MK, Zdraveski Z, Treer JR, Waldrop SL, et al. (1995) Direct demonstration of cytokine synthesis heterogeneity among human memory/ effector T cells by flow cytometry. Blood 86: 1408-1419.

25. Hamann D, Baars PA, Rep MH, Hooibrink B, Kerkhof-Garde SR, et al. (1997) Phenotypic and functional separation of memory and effector human CD8+ $T$ cells. J Exp Med 186: 1407-1418.

26. Hambleton S (2011) Editorial commentary: a better grip: T cells strengthen our hand against influenza. Clin Infect Dis 52: 8-9.

27. Madhun AS, Haaheim LR, Nilsen MV, Cox RJ (2009) Intramuscular Matrix-Madjuvanted virosomal $\mathrm{H} 5 \mathrm{~N} 1$ vaccine induces high frequencies of multifunctional Th1 CD4+ cells and strong antibody responses in mice. Vaccine 27: 73677376 .

28. Madhun AS, Haaheim LR, Nostbakken JK, Ebensen T, Chichester J, et al (2011) Intranasal c-di-GMP-adjuvanted plant-derived H5 influenza vaccine induces multifunctional Th1 CD4(+) cells and strong mucosal and systemic antibody responses in mice. Vaccine 29: 4973-4982.

29. Purwar R, Campbell J, Murphy G, Richards WG, Clark RA, et al. (2011) Resident memory $T$ cells $(T(R M))$ are abundant in human lung: diversity, function, and antigen specificity. PLoS One 6: e16245. 